\title{
Genotypic Detection of Extended Spectrum Beta Lactamases from Selected Bacterial Isolates in the Specialist Hospital Sokoto, Nigeria
}

\section{*11 UNGO-KORE, HY; ${ }^{1}$ BULUS, MW; ${ }^{1}$ NUHU, T; ${ }^{1}$ OLOWOOKERE, A; ${ }^{1}$ SAADATU, MJ; ${ }^{1}$ ALHASAN, MA; ${ }^{1}$ IBRAHIM, AO; ${ }^{2}$ SHUAIBU, AS}

\author{
${ }^{l}$ Department of Pharmaceutics and Pharmaceutical Microbiology, ${ }^{1}$ Department of Pharmaceutical and Medicinal Chemistry, ${ }^{1}$ Department \\ of Clinical Pharmacy and Pharmacy Practice, Usmanu Danfodiyo University, Sokoto, Nigeria \\ ${ }^{2}$ Department of Pharmaceutics and Pharmaceutical Microbiology, Ahmadu Bello University, Zaria, Nigeria. \\ *Corresponding Author Email: ungokorehussain@yahoo.com,nuhu.tanko@yahoo.com (+234(0)8033647999)
}

\begin{abstract}
There are numerous reported cases of extended spectrum beta lactamases (ESBLs) producing Enterobacteriaceae in Nigeria, with little effort done on the molecular detection. Epidemiological studies around the world have investigated the prevalence of ESBL-producing enterobacteriaceae and they have seen multiple mechanisms of drug-resistance. Our study was designed to detect ESBLs genes such as CTX-M, SHV, and TEM using PCR from clinical isolates in a tertiary hospital in Sokoto metropolis. Clinical isolates from the Microbiology laboratory of the tertiary hospital was collected for 3 months. These isolates were identified using standard microbiological methods. They were tested against 8 antibiotics using the modified Kirby Bauer disc diffusion method. Multidrug resistant isolates were screened for ESBL production, and further confirmed by the Double Disc Synergy Test (DDST). Genotypic confirmation was carried out using multiplex Polymerase Chain Reaction (PCR). A total of 47 isolates made up of $21 \mathrm{E}$. coli $(44.6 \%), 13$ Klebsiella spp (27.6\%), 7 Salmonella spp (14.9\%), 5 Proteus mirabilis (10.6\%), and 1 Enterobacter spp (2.1\%) were obtained from urine, stool, and wound swab. Out of the 47 isolates, (45) 95.7\% were multidrug resistant. Twenty-five (53.2\%) were potential ESBL producers, while only 5 $(20.0 \%)$ were confirmed phenotypically using a DDST. PCR results revealed 4 out of 5 of the isolates were possessing ESBL genes. It also revealed that 3 isolates co-produce TEM and SHV at $403 \mathrm{bp}$ and 293bp respectively. Only 1 isolate produced CTX-M gene at 569bp. The prevalence of ESBL production in the Gram negative enterobacteriaceae in our study did not indicate a high prevalence as reported by some studies in Sokoto and Northwest Nigeria.
\end{abstract}

DOI: https://dx.doi.org/10.4314/jasem.v23i8.23

Copyright: Copyright $($ C) 2019 Ungo-Kore et al. This is an open access article distributed under the Creative Commons Attribution License (CCL), which permits unrestricted use, distribution, and reproduction in any medium, provided the original work is properly cited.

Dates: Received: 25 June 2019; Revised: 13 August 2019; 19 August 2019

Keywords: Molecular detection, ESBLs, Clinical isolates, PCR

Polymerase chain reaction (PCR) is a valuable technique in the diagnosis of bacterial infection. Molecular methods might be superior to bacterial culture in providing the etiologic diagnosis (Morgenstern et al., 2018). Another advantage of bacterial PCR is connected to its broad-range nature since rare or unexpected pathogen can be found. A comparison to culture results reveals difficulties in detecting gram positive species and Mycobacterium by PCR (Mirsaeidi et al., 2014). Production of ESBLs is one of the most prevalent resistance mechanisms in Gram-negative bacilli. Initially, ESBLs were predominantly described in K. pneumoniae and E. coli strains, but recently the enzymes were found in other genera of the Enterobacteriaceae family (Shaikh et al., 2015). Extended Spectrum Beta Lactamases (ESBLs) in human medicine first came to prominence following the introduction of the extended-spectrum cephalosporins (Rebbah et al., 2018). In the 1980's, the term was applied to mutants of the already common plasmid mediated $\beta$-lactamases such as SHV and TEM. One of the first detected, which originated in France, was TEM-3 ESBL which had been presumed to have been selected in the human host following the introduction and use of cefotaxine. Both of the 'parent' genotypes of this $\beta$-lactamase genes blaTEM-1 blaTEM-2 were widely disseminated amongst medically important members of Enterobacteriaceae and Acinetobacter spp. and it seemed logical (Mendonça, 2009). The extended spectrum form of the $\beta$-lactamase would become much more widely disseminated throughout the world under the selective pressure of the use of third generation cephalosporins (Ahlstrom et al., 2018). This happened to a degree in that particularly some variants of TEM, such as TEM-10, TEM-24 and SHV-12, became widely distributed, particularly amongst Klebsiella .spp. in hospital settings, but never reached high levels of prevalence (usually $<20 \%$ ). There also was never a significant spread of these genes into the more general 
environmental and commensally pool of Enterobacteriaceae, even in countries where ESBL is predominant (Iredell et al., 2016).

Phenotype rates were reported to be very high, such as in China and India. The initially named MEN-1 ESBL from an E. coli isolate characterized in France from a patient in Italy (1990) was found to be identical to CTX-M-1 from an E.coli isolated from a child in Germany (1989) (Hawkey, 2017) . The objective of this paper is the genotypic detection of extended spectrum beta lactamases from selected bacterial isolates obtained from Specialist Hospital Sokoto, Nigeria.

\section{MATERIALS AND METHODS}

Sample Collection: The isolates were obtained from the Microbiology laboratory in the Specialist Hospital Sokoto and were taken to the department of Pharmaceutics and Pharmaceutical Microbiology Laboratory Usmanu Danfodiyo University Sokoto for further analysis.

Preliminary Test for ESBL Production: The isolates were screened for ESBL production using cefotaxime $(30 \mu \mathrm{g})$ and the ceftazidime $(30 \mu \mathrm{g})$ discs as described by CLSI (2015).

Confirmatory Test for ESBL Production: Confirmation of ESBL phenotype was performed for 47 clinical samples obtained from blood, urine, stool, and saliva by Double Disc Synergy Test (DDST) using antibiotic discs containing two $3^{\text {rd }}$ generation Cephalosporin (CTZ, 30ug and CTX, 30ug) and Augumentin(AMC, 30ug). Using a sterile needle, CTZ and CTX were placed at a distance of $20 \mathrm{~mm}$ center to center from AMC on Mueller Hinton Agar. The plates were then incubated at $37^{\circ} \mathrm{c}$ for 24 hours after which they were examined for an extension of the edge of zone of inhibition of antibiotic disc towards the AMC. Enhancement of zone of inhibition of either or both the CTZ and CTX towards the AMC disc is indicative of ESBL production (EUCAST, 2014)
Genotypic detection of ESBL: Isolates preparation: The materials used in the preparation was sterilized using the autoclave, then the peptone water was prepared by weighing $1.275 \mathrm{~g}$ of solid peptone which was dissolved in $50 \mathrm{mls}$ distilled water inside a beaker of $100 \mathrm{ml}$ capacity by stirring on a water bath until it completely dissolved. Then it was cover with an aluminum foil and was sterilized in the autoclave at $121^{\circ} \mathrm{C}$ for $15 \mathrm{mins}$. Then $5 \mathrm{mls}$ sterile syringe was used to aseptically transfer $5 \mathrm{mls}$ of the peptone water into five different test tubes and covered. Then the inoculums which were stored in the refrigerator was aseptically transferred into each of the test tubes already labeled with sample 1-5 where 1,3 and 5 are Klebsiellas, 2 is Proteus mirabilis, and 4 is E. coli. Then they were incubated at $37^{\circ} \mathrm{C}$ for $24 \mathrm{hrs}$.

DNA Extraction: An overnight suspension of the test bacteria was prepared by transferring a loop full of the isolates into a test tube containing $2 \mathrm{mls}$ of peptone water. The suspension was centrifuged at $6000 \mathrm{rpm}$ for 5 minutes then, the supernatant was discarded and the sediments (cells) were transferred into a $1.5 \mathrm{mls}$ microfuge tube. Then, the cells were re suspended in a $50 \mu \mathrm{l}$ of nuclease free water and the tubes were transferred into a water bath and boiled at $95^{\circ} \mathrm{C}$ for 30 minutes. Then the tubes were allowed to cool and were centrifuged at $13000 \mathrm{rpm}$ for 5 minutes at $4^{\circ} \mathrm{C}$, then the supernatants were transferred to a nuclease free $0.5 \mathrm{ml}$ microfuge tubes and the extracted DNA were stored at $-20^{\circ} \mathrm{C}$ until when required for the PCR (Central Research Laboratory, 2018).

Multiplex PCR of ESBL: A total of $25 \mu 1$ PCR reaction mix containing $5 \mu \mathrm{l}$ of the DNA template was used. $12.5 \mu 1$ of Biorad mixer, $4.5 \mu$ lof nuclease free water and $0.5 \mu \mathrm{l}$ each of the following primers were multiplexed The PCR primers were transferred to a Biorad 9700 thermocycler and subjected to initial denaturation at $94^{\circ} \mathrm{C}$ for 3 minutes. The PCR was carried using the procedure below

Table 1. Primer sequences

\begin{tabular}{llll}
\hline Primers & Tm & Nucleotide Sequence (5'-3') & Size (bp) \\
\hline SHV -F & 60 & CGCCTGTGTATTATCTCCCT & \\
SHV-R & 62 & CGAGTAGTCCACCAGATCCT & 293 \\
TEM-F & 60 & TTTCGTGTCGCCCTTATTCC & \\
TEM-R & 62 & ATCGTTGTCAGAAGTAAGTTGG & 403 \\
CTX-M-F & 60 & CGCTGTTGTTAGGAAGTGTG & \\
CTX-M-R & 62 & GGCTGGGTGAAGTAAGTGAC & 569 \\
\hline
\end{tabular}

UNGO-KORE, HY; BULUS, MW; NUHU, T; OLOWOOKERE, A; SAADATU, MJ; ALHASAN, MA; IBRAHIM, AO; SHUAIBU, AS 
DNA Amplification: Amplification was be carried out in an Eppendorf thermal cycler, according to the following thermal and cycling conditions for the TEM, SHV and CTX-M gene. Initial denaturation at $94^{\circ} \mathrm{C}$ for 3 minutes, denaturation at $94^{\circ} \mathrm{C}$ for 45 seconds of 35 cycles, annealing at $60^{\circ} \mathrm{C}$ for $30 \mathrm{sec}$ of 35 cycles, extension at $72^{\circ} \mathrm{C}$ for 3 minutes of 35 cycles and final extension at $72^{\circ} \mathrm{C}$ for 2 minutes (Vincent et al., 2018)

Agarose Gel Electrophoresis: After ensuring that the electronic digital balance was calibrated, $1.5 \mathrm{~g}$ of Agarose powder was weighed and dissolved in $100 \mathrm{ml}$ of distilled water and was transferred into the water bath for complete dissolution. Then the molten agar was allowed to cool to about $50^{\circ} \mathrm{C}$ and $5 \mu 1$ of ethidium bromide was added and swirled to mix. The gel was poured into a wide Biorad gel casting unit, and was allowed for 30minutes to solidify and transferred into an agarose gel tank and was flooded with $500 \mathrm{mls}$ of $0.5 \mathrm{x}$ TBE buffer. Then $10 \mu 1$ of the standard was mixed with a $6 \mathrm{x}$ loading dye and loaded in each allocated wells. The tank was closed and the electrophoresis power pack was programmed to run at 10 volts for $30 \mathrm{~min}$. then the gel outcome was viewed using Biorad gel imager. The result was obtained with reference to the base pairs of both forward and reverse base pairs (Westermeier, 2016).

\section{RESULTS AND DISCUSSION}

ESBL Percentage Distribution: The result in fig1 shows that out of the 47 isolates collected, only 5 were confirmed to produce ESBL, showing a prevalence of $11 \%$ in the Specialist Hospital Sokoto. The highest prevalence was found in Klebsiella spp (3 out of 5) followed by Proteus mirabilis (1 out of 5), and E. coli (1 out of 5).

\section{Percentage Distribution of ESBL and Non ESBL Producers}

From the result obtained in fig 2, $80 \%$ of the processed samples were found to be positive for ESBL which was in line with to Nuhu et al., 2016 who concluded that the ESBL producing uro- pathogen in Sokoto metropolis was $(66 \%)$ while the remaining $20 \%$ was negative showing a high percentage reliability of the phenotypic detection. The result obtained from plate 2 shows a gel image of the amplified genes in which the CTX-M, was amplified at 569bp and happens to be the largest, followed by TEM amplified at $403 \mathrm{bp}$, then SHV amplified at 293bp. The M was the marker and the $\mathrm{NC}$ was for the negative control. There was $11 \%$ prevalence of ESBL obtained in the Specialist Hospital Sokoto, which was in line with the $15.4 \%$ obtained by Tanko et al in 2015 and far from Kaduna state that was reported to have confirmed ESBL (37.5\%) by Shetu et al in 2017 from snacks.

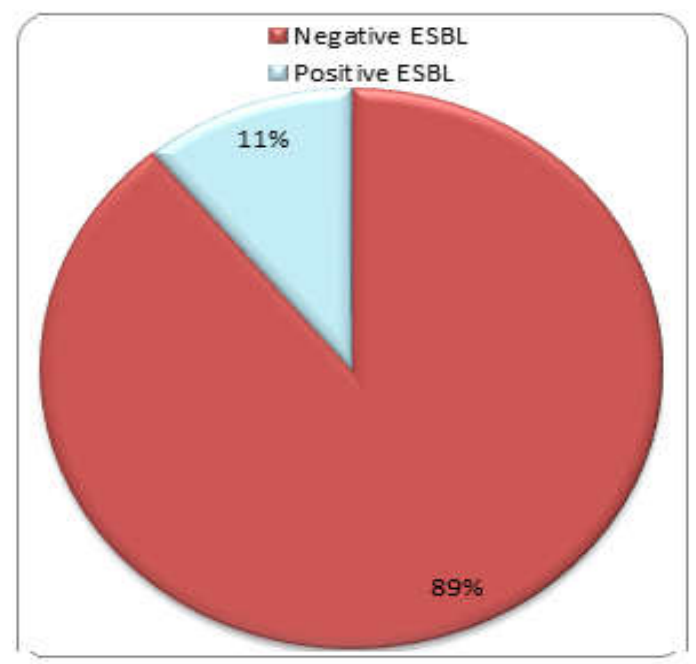

Fig. 1: Percentage distribution of ESBLs producing strains using Cefotaxime

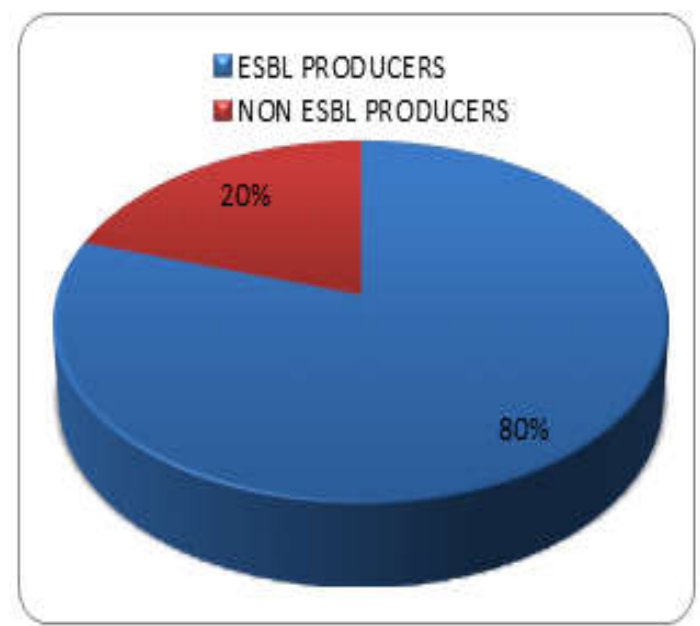

Fig.2 Percentage distribution of ESBL and non ESBL producer for TEM, SHV, or CTX-M

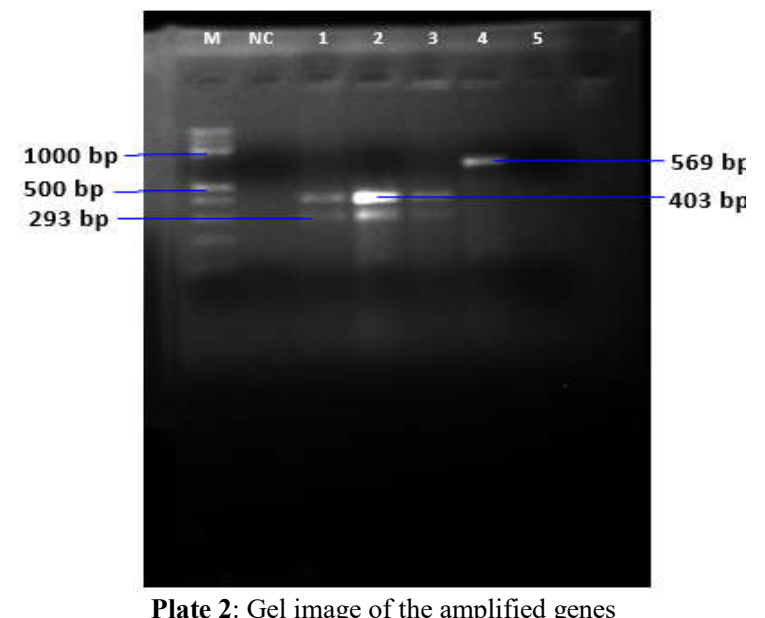

M: marker, $N C=$ negative control, $1=$ sample E1, $2=$ sample E2, $3=$ sample E3, 4= sample E4, 5= sample E5, SHV - 293bp, TEM403bp, CTXM- 569bp. E1, E3 and E5 are Klebsiella spp while $E 2=$ Proteus mirabilis and $E 4=$ Escherichia coli

UNGO-KORE, HY; BULUS, MW; NUHU, T; OLOWOOKERE, A; SAADATU, MJ; ALHASAN, MA; IBRAHIM, $A O ; S H U A I B U, A S$ 
Adeluola, et al., 2018 carried a research on the detection, inhibition and molecular analysis of multidrug resistant aerobic gram-negative clinical isolates from a tertiary hospital in Nigeria in which they obtained a result of $12 \%$ of ESBL genes. The remaining $89 \%$ were negative which signifies that majority of the population sampled were free from the ESBL production but a total clearance is expected because the $11 \%$ can spread over time to the rest of the population. From the multiplexed PCR results obtained in fig2 the different amplicon size of 293bp for SHV-F (CGCCTGTGTATTATCTCCCT), SHVR(CGAGTAGTCCACCAGATCCT), 403bp for TEM-F(TTTCGTGTCGCCCTTATTCC), and 569bp for CTX-M primers for both forward and reverse Oligonucleotides was observed that sample 1,2 and 3 have two bands of amplicons of 293bp and $403 \mathrm{bp}$ which signifies that the primers that amplified the gene for ESBL were SHV and TEM respectively while sample 4 has only one band of 569bp which shows that it was amplified by CTX-M primer and sample 5 has no band signifying that there is no gene coding for ESBL or the specific primer that can amplify the gene was not used for the experiment, but $80 \%$ of the isolates where ESBL producers, while $20 \%$ of the sample which was just 1 was found to be non ESBL producer.

Conclusion: The prevalence of ESBLs in the hospital samples collected was $11 \%$ and there is a high risk of it rising to a higher percentage due to the fact that patient and health workers could be a medium of transferring some of this resistant organism. The percentage of the detected ESBL producers was $80 \%$ which means that the phenotypic methods of identification could be reliable.

\section{REFERENCES}

Adekunle, O.O (2012). Mechanisms of Antimicribial Resistance in Bacteria, General approach. International Journal of Pharmacy, Medicine and Biological Sciences, 1 (2), 166-187.

Adeluola, A. O., Oyedeji, K. S., Mendie, U. E., Johnson, J. R., Porter, J. R. (2018). Detection, inhibition and molecular analysis of multidrug resistant aerobic gram-negative clinical isolates from a tertiary hospital in Nigeria. African Journal of Biomedical Research, 21(1), 15-21.

C. L. S. I (2011). Performance standards for antimicrobial susceptibility testing: 20th international supplement m 100-S20 -u, 30,15

Clinical and Laboratory Standards Institute. (2015). Performance Standards for Antimicrobial
Susceptibility Testing; Sixteenth Informational Supplement. Wayne, PA, US.: CLSI Document M100-S16. CLSI.

European Committee on Antimicrobial Susceptibility Testing. (2014). EUCAST.

Iredell, J., Brown, J., and Tagg, K. (2016). Antibiotic resistance in Enterobacteriaceae: mechanisms and clinical implications. Bmj, 352, h6420.

Hawkey, P. (2017). The epidemiology and molecular evolution of the CTX-M betalactamases (Doctoral dissertation, University of Birmingham).

Mirsaeidi, M., Farshidpour, M., Ebrahimi, G., Aliberti, S., and Falkinham III, J. O. (2014). Management of nontuberculous mycobacterial infection in the elderly. European journal of internal medicine, 25(4), 356-363.

Morgenstern, C., Renz, N., Cabric, S., Perka, C., Trampuz, A. (2018). Multiplex Polymerase Chain Reaction and Microcalorimetry in Synovial Fluid: Can Pathogen-based Detection Assays Improve the Diagnosis of Septic Arthritis? The Journal of rheumatology, jrheum-180311.

Mendonça, N. R. F. D. (2009). Molecular diversity of blaz genes in Klebsiella pneumoniae and Escherichia coli isolates.

Nuhu, T., Bolaji, R. O., Olayinka, B. O. (2016). Prevalence And Antimicrobial Susceptibility Of Extended Spectrum Beta-Lactamase (Esbl) Producing Gram-Negative Uropathogens In Sokoto, Nigeria. Nigerian Journal of Pharmaceutical Research, 11(1), 59-65.

Rebbah, N., Messai, Y., Châtre, P., Haenni, M., Madec, J. Y., Bakour, R. (2018). Diversity of CTX-M extended-spectrum $\beta$-lactamases in Escherichia coli isolates from retail raw ground beef: first report of CTX-M-24 and CTX-M-32 in Algeria. Microbial Drug Resistance, 24(7), 896908.

Shaikh, S., Fatima, J., Shakil, S., Rizvi, S. M., Kamal, M. A. (2015). Antibiotic Resistance and Extended Spectrum Beta-Lactamases: Types, Epidemiology and Treatment. Saudi Journal of Biological Sciences, 22, 90-101.

Shaikh, S., Fatima, J., Shakil, S., Rizvi, S. M. D., \& Kamal, M. A. (2015). Antibiotic resistance and extended spectrum beta-lactamases: Types, 
epidemiology and treatment. Saudi journal of biological sciences, 22(1), 90-101.

Shetu, E. D., Yushau, M., Uba, A., Sonny, O. J., \& Usman, E. I. (2017). Detection of Extended Spectrum B-Lactamases Among Bacteria Isolated from Snacks Sold Within Kaduna State Metropolis Northwest Nigeria. Medicine, 1(3), 92-97.

Tanko, T. (2017). Phenotypic detection of extended spectrum Beta-Lactamases(ESBL) produced by Gram negative Uropathogens in Sokoto metropolis. Sokoto Journal of Medical Laboratory Science, 106-112.
Vincent, J. P., Komaki-Yasuda, K., Iwagami, M., Kawai, S., Kano, S. (2018). Combination of PURE-DNA extraction and LAMP-DNA amplification methods for accurate malaria diagnosis on dried blood spots. Malaria journal, 17(1), 373 .

Westermeier, R. (2016). Electrophoresis in practice: a guide to methods and applications of DNA and protein separations. John Wiley and Sons. 